International Journal of Engineering \& Technology, 7 (4.33) (2018) $487-490$.
International Journal of Engineering \& Technology
WPC
Website: www.sciencepubco.com/index.php/IJET
Research paper

\title{
3D Robot Vision System through 2D Shape Based Matching Using Gaussian Smoothing for Gluing Application
}

\author{
Mohamad Haniff Harun ${ }^{1,2 *}$, Mohd Shahrieel Mohd Aras ${ }^{2}$, Mohd Firdaus Mohd Ab Halim ${ }^{1,2}$, Khalil Azha Mohd \\ Annuar ${ }^{1,2}$, Arman Hadi Azahar ${ }^{1,2}$, Muhamad Faizal Yakub ${ }^{1,2}$, Amar Faiz Zainal Abidin ${ }^{1}$ \\ ${ }^{1}$ Faculty of Electrical and Electronic Engineering Technology, Universiti Teknikal Malaysia Melaka (UTeM), \\ Hang Tuah Jaya, 76100 Durian Tunggal, Melaka, Malaysia \\ ${ }^{2}$ Centre for Robotic and Industrial Automation (CeRIA), Faculty of Electrical Engineering, Universiti Teknikal Malaysia Melaka, \\ Hang Tuah Jaya, 76100 Durian Tunggal, Melaka, Malaysia \\ *Corresponding author E-mail: haniff@utem.edu.my
}

\begin{abstract}
This investigation is solely on the adaptation of a vision system algorithm to classify the processes to regulate the decision making related to the tasks and defect's recognition. These idea stresses on the new method on vision algorithm which is focusing on the shape matching properties to classify defects occur on the product. The problem faced before that the system required to process broad data acquired from the object caused the time and efficiency slightly decrease. The propose defect detection approach combine with Region of Interest, Gaussian smoothing, Correlation and Template Matching are introduced. This application provides high computational savings and results in better recognition rate about $95.14 \%$. The defects occur provides with information of the height which corresponds by the z-coordinate, length which corresponds by the y-coordinate and width which corresponds by the $\mathrm{x}$-coordinate. This data gathered from the proposed system using dual camera for executing the three dimensional transformation.
\end{abstract}

Keywords: Correlation; Gaussian Smoothing; Region of Interest; Template Matching, Vision System.

\section{Introduction}

Vision algorithm system consists mainly, seven essential procedures which are image collection procedure, pre-processing of the image procedure, image segmentation procedure, low-level feature extraction procedure, high level feature extraction by grouping or mapping procedure, procedure related to image classification/identification and lastly, procedure related to image interpretation [1]. In determining the image, it is important to use a simple algorithm but give the best impact according to the given task. The best algorithm is capable of delivering more accurate and fast results. For instance, an object can be identifying or recognize by implementing the zoning/regional algorithm such as treating the image based on colour, shape and texture as the input for the algorithm [2]. Implementation of the voting-based method is done to identify based on object locations, scale and support. Area of interest have free highlights that make its basic amid the recognizable proof stage since they give data identified with shape and scale information of the articles normally. They are mildly affected by background clutter.

Most of vision approaches which provide a solution by interpreting the image or object in image processing by capturing information related to region of interest [2], interpreting features of the edge-based [3], extracting features [4], analyzing the context of the shape [5], low distortion correspondences [6] and etc. Alternate scientists for the most part centered around HALCON Application for Shape-Based Coordinating that gives additional data in vision characteristic [7-10]. This paper features principally on the proposed procedure engaged with a shape based matching (SBM) calculation with extra of broadened Area of Interest (ROI) that satisfies SBM to regards a picture as a solitary model and after that find the model with sub-pixel precision level. The fundamental approach of defect matching by implementing algorithm which match the shape of the extended region of interest introduced by [11].

In the region of Machine Vision framework, many techniques have been concentrated seriously for the most part on the deformity of welding and surface with a lot of machine vision calculations are accessible. As being presented by [12], the creator utilizes highlight separated as a technique to limit the normal for a picture to a straightforward calculation which uses the perceptron model to decide the deformities as indicated by the information assembled from the extraction approach. Displaying of the welding deformity is fundamental as an extreme body structure in getting the best acknowledgment rate for imperfection discovery calculation. A Gaussian distribution curve can be used as a tool to make approximation on the other types of algorithm being described by [13] where the development based on the assumption that a line profile of defect less weld image. The line profile varieties of weld imperfection brought about by deformities are classified into three distinct examples. By according to the result, the development of defect inspection system achieved successful recognition rate of $95 \%$ based on 24 tested images and consists of 61 defects.

According to [14], a new method to texture defect detection based on a set of optimized filter is implemented. An excellent framework to deal with multi-scale defects is provided by the wavelet transform. The proposed approach proposes the wavelet packet to be decompose into three levels where the first, second and third level will have four, 16 and 64 square parts. Therefore, the filter applied in higher wavelet those results in easier to detect defect as it requires only a small part of spectrum analysis. Gabor filters as 
main approach for texture defect detection, Fourier series analysis, adaptive two-dimensional filter, fuzzy clustering method [15-18] are the other research that contributes on the defect inspection system. Among the other related researches are being introduced by [19] in which a review of recent techniques that being used in surface inspection using computer vision and image processing techniques for image checking processes and schemes form results that can distinguish features extracted from normal and damaged areas

\section{Proposed System}

This research is design a system that can be adopted into industrial automation for gluing process of windscreen car. Adapting to the environment, for example, lighting condition, scaling and pivoting of the item is among the pre - imperative of the framework development. Pyramid object developed from plain cardboard is created based on the shape object that has a contour that involves height, length and width. To get the data from the Defect Shape Matching (DSM), the plain cardboard is design exactly like pyramid whether the system recognized it or not. Each time they got data from system through Defect Shape Pointing (DSP), an arm robot named KUKA provides gluing service. To generate information $\mathrm{x}, \mathrm{y}$ and $\mathrm{z}$ coordinate, two cameras will be placed as an ideal position and angles. In Defect Inspection Monitoring (DIM), there are three types of defect that is worried to be occurring such as gap defect, bumper defect and bubble defect. Result obtained from DIM will decide whether the object is successful gluing or needs to be corrected through Correction of Defect (CoD). Fig. 1 shows the overall system of this research.

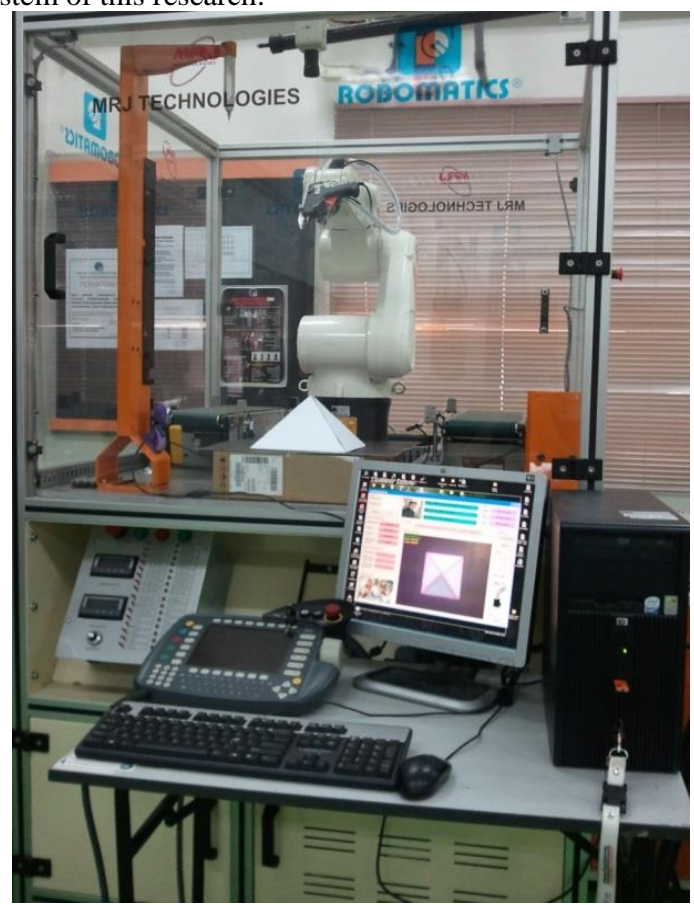

Fig. 1: Overall System Design

\subsection{Three Dimensional Representation (z-Data Trans- formation)}

Gluing procedure includes $\mathrm{x}, \mathrm{y}$ and $\mathrm{z}$ hub in deciding the situation as per the working space of the KUKA robot arm. This camera serves significantly in distinguishing the item that need to go for gluing procedure. This exploratory framework is being redesigned subsequently updated whereby applying another camera before the item so as to restore the z-coordinate in the picture. The technique utilized are to acquire the 3D object acknowledgment characterizing as $\mathrm{x}, \mathrm{y}$ and $\mathrm{z}$-pivot by utilizing assessment work so as to decide better position and introduction for camera situation. Accord- ing to [20], the technique collinear as Fig. 2 demonstrates the proposed strategy by utilizing two cameras from the specialist.

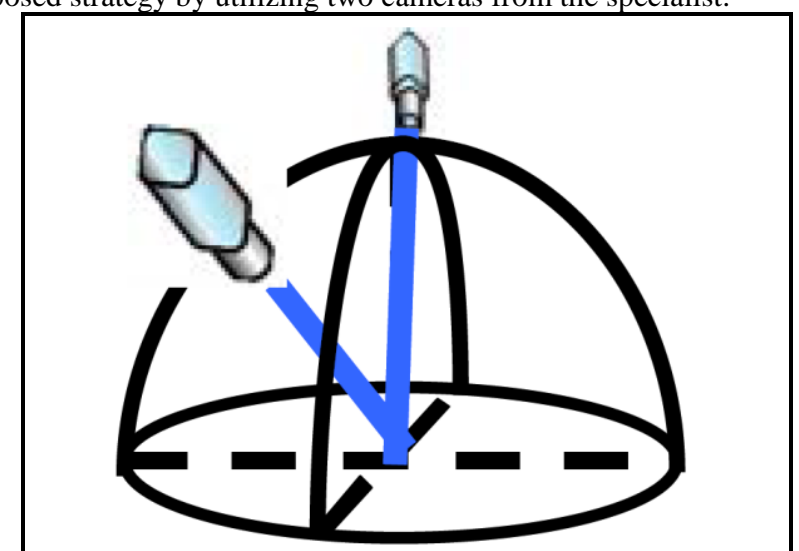

Fig. 2: Placement of Two Camera of 3D Recognition

With the direction of visual in Fig. 2, this design can repeat the 3D change as per the model format that being made in the framework. The estimation of the camera considers all the side of the article which will be process in deciding the careful estimation of change with the first. From this application, the improvement of new proposed strategy by utilizing two cameras with various positions and orientations in reason for deciding $\mathrm{x}, \mathrm{y}$ and $\mathrm{z}$-coordinate for further procedure in this process. The redesign of this new strategy being proposed is utilized in making a $\mathrm{z}$-coordinate as indicated by the $\mathrm{x}$ and $\mathrm{y}$-pivot from pictures caught. Fig. 3 demonstrates the new proposed strategy created in this process.

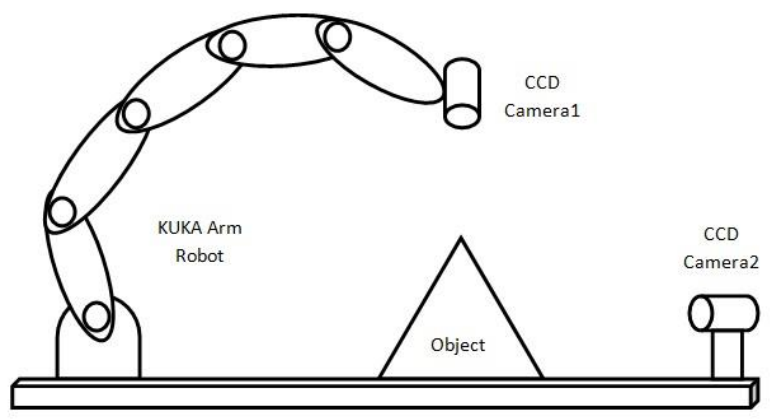

Fig. 3: Proposed Position of two Camera Placement in 3D

\subsection{Matching in Proposed Vision Algorithm}

The main idea of this investigation is to determine faulty of the robot upon finishing the task to execute the gluing task before the particular specification given by the vision sensor. Too many techniques can be used to build up a framework that required insightful in identifying defects, but there is a diversity of different algorithm concepts that carry its own weight of yet to be known strengths and weaknesses. Shape-based coordinating calculation was picked to be utilized in this exploration from these calculations for the most part because of clinging to the strain of the inspection necessity on the investigation of a steady and dreary sort of picture. As for the supporting reasons of this shape - based matching algorithm chosen also due to numerous applications that might occur hence shape-based matching which take the best of the outline edges for this investigation since everything has a shape. Fig. 3 demonstrates the task improvement of deformity review framework by utilizing two cameras set at best and front position for identifying all the three directions known as stature (zaxis), width (x-axis) and length (y-axis). 


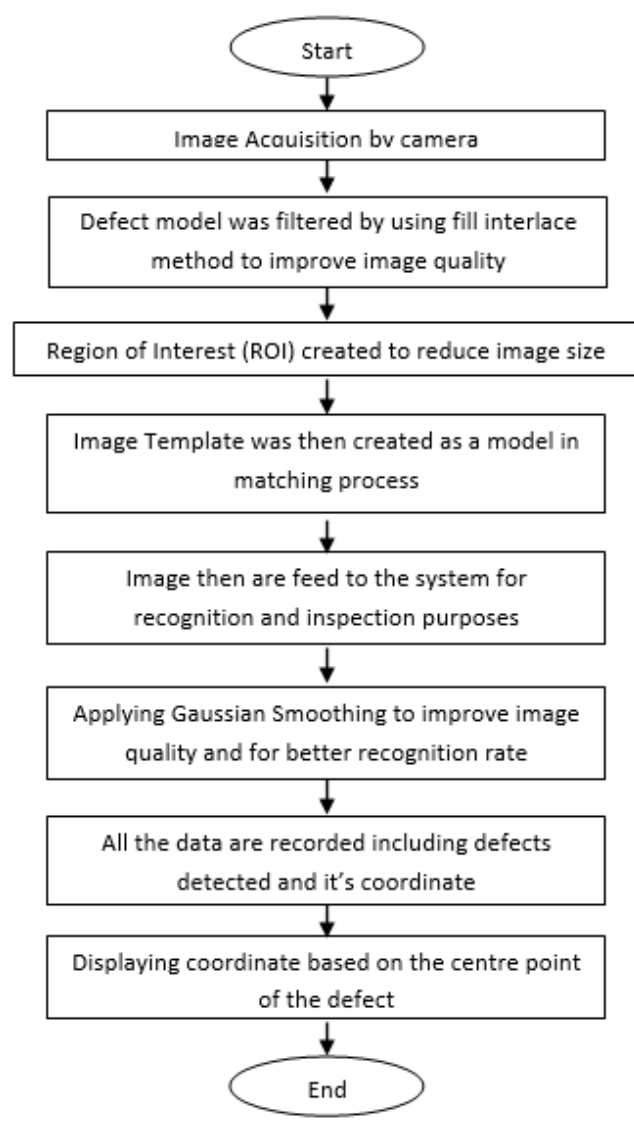

Fig. 4: Proposed System for Defect Inspection System

HALCON programming which is the essential extent of this process gives a wide scope of library that will helps for the most part on picture preparing calculation. It can be manipulated based on the task that meets system requirement. The basic of shape matching application required 2 phases which are training and recognition stage. During the training stage, whole data chosen for training will be run using the shape detection library, the prepared picture will be put away in memory as template that later will be helpful for the recognition stage. Pictures at the recognition stage are then bolstered to the proposed methodology so as to be coordinated against the template that was made in the training stage. In light of this framework, the proposed framework appeared in Fig. 4 is created dependent on the undertaking required in deciding the glue defects once the glue application is connected. Once the template has been created through training phase, template matching takes placed. An algorithm that compares portions of images against one another is defined as template matching. Beforehand, the template of a specimen must be characterized first before utilized it in perceive comparable items in source picture [21].

\section{Results and Discussion}

The capability of the proposed vision calculation was the adaptability of the calculation to suit changes stage by stage. Fifty tried pictures are utilized to test the strength of the framework in characterizing defects dependent on the tried pictures. The tried pictures are intentionally loaded up with each of the three kinds' model imperfections that are as of now being utilized in preparing stage and encouraged into the framework for acknowledgment purposes. Each imperfection that are coordinate through the framework will furnish with its own trademark dependent on the grid arranges as per the pixel organizes in the framework. This is fundamental as the undertaking continuing in redressing every one of the imperfections after the coordinating procedure carried out its responsibility. Recognition stage comprises of a KUKA arm robot to perform change on the deformities to guarantee that glue is fit as a fiddle.

Identifying with the Fig. 5, it very well may be seen that the consequence of imperfection detection demonstrates an aggregate of 8 deformities and every last bit of it has been recognized through the proposed framework. Every one of deformities gives their very own area for further procedure. For second tried picture as appeared in Fig. 6, demonstrates that there is one imperfection that isn't being recognized due to the splendid light that irritates the framework from complete its undertaking. The form of the imperfections has been disposed of by the brilliance itself and the framework remembered it as an ideal gluing line.

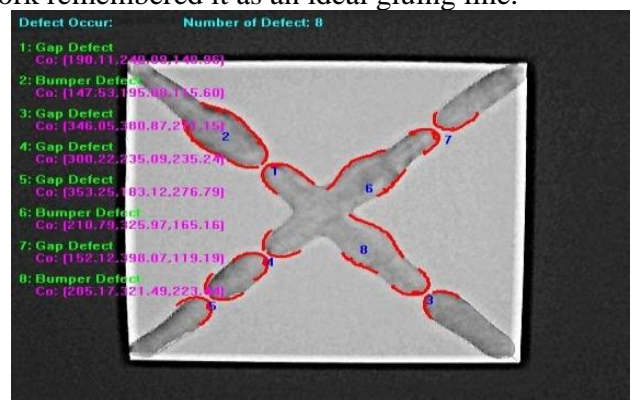

Fig. 5: First Tested Images

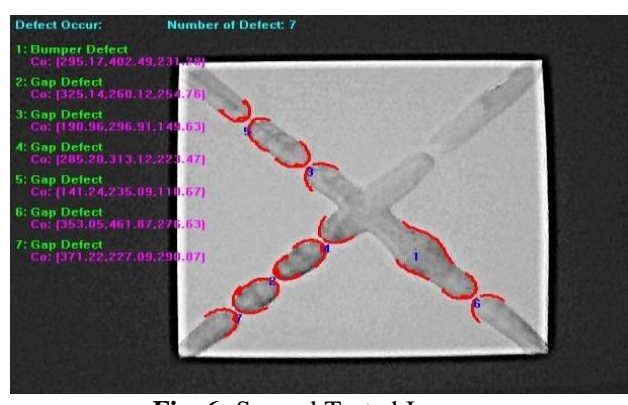

Fig. 6: Second Tested Images

The point of this paper is to exhibit an adaptable visual framework for shape based matching. Through this paper, expansion of highlight extraction, Gaussian smoothing, template creation and format coordinating are being proposed. The proposed technique approach being confirmed utilizing the exploratory outcomes. Three deformities models and their correcting tests are utilized in this process to analyze this methodology. 10 pictures are being tried multiple times including 720 imperfections has tried the framework to decide framework precision and proficiency in recognizing paste deformity along gluing line. Each tried picture has its very own imperfection to be perceived by this framework. Every one of the information are recorded into Table 1.

Table 1: Recognition Rate for Proposed Defect Inspection System

\begin{tabular}{|c|c|c|c|}
\hline Number of Test & Total Defect & $\begin{array}{c}\text { Defect } \\
\text { Detected }\end{array}$ & $\begin{array}{c}\text { Average Defect } \\
\text { Detected (\%) }\end{array}$ \\
\hline 1 & 72 & 69 & 95.83 \\
\hline 2 & 72 & 68 & 94.44 \\
\hline 3 & 72 & 67 & 93.06 \\
\hline 4 & 72 & 70 & 97.22 \\
\hline 5 & 72 & 68 & 94.44 \\
\hline 6 & 72 & 69 & 95.83 \\
\hline 7 & 72 & 67 & 93.06 \\
\hline 8 & 72 & 68 & 94.44 \\
\hline 9 & 72 & 69 & 95.83 \\
\hline 10 & 72 & 70 & 97.22 \\
\hline Total & 720 & 685 & 95.14 \\
\hline
\end{tabular}

Table 1 arranges the aftereffect of deformity coordinating by utilizing 10 tried pictures. Inferring to the outcomes acquired the accurately perceive at the rate of $95.14 \%$ dependent on three model imperfections made through the framework. This result is compared with literature review of 'A Method for Recognition of Defects in Welding Lines' [12] where it considered the problem in 
detecting welding defects in welding lines where the past researchers put an effort on more complex algorithm or limited in efficiency. The main objectives of this research is to classify 6 types of defect might occur in welding lines such as air hole, crack, strip inclusion, round inclusion, lack of penetration and metal inclusion. To define the capable of this algorithm, the suggested computer auto-recognized welding defect is being put forward. To evaluate the defect detection capabilities, the algorithm is tested with 500 welding defect consists of all six types of defects. The results of this experiments represents defect detection of each defect's type but overall this system able to generate $94.3 \%$ success rate in classifying defect's type. Although the experiments setup, objective and methods are not entirely similar but basically the goal of each experiment is to develop a vision system in inspection for defect detection in industry. The technique in inspection of gluing application even though is still new is still the same with welding application but just differs in how to analyze characteristic of each defects. Table 2 below shows the result compared from both research.

\begin{tabular}{|c|c|c|}
\multicolumn{1}{|c|}{ Table 2: Comparison of Results } \\
\begin{tabular}{|c|c|c|}
\hline & $\begin{array}{c}\text { Weld Defect } \\
\text { Detection }\end{array}$ & $\begin{array}{c}\text { Glue Defect } \\
\text { Detection }\end{array}$ \\
\hline Number of Samples & 500 samples defect & 726 samples defect \\
\hline Variation of Samples & 6 types & 3 types \\
\hline $\begin{array}{c}\text { Correct Recognition } \\
\text { Rate }(\%)\end{array}$ & $94.3 \%$ & $95.14 \%$ \\
\hline Error Rate $(\%)$ & $5.7 \%$ & $4.86 \%$ \\
\hline
\end{tabular}
\end{tabular}

From the results, it can be seen that the system's efficiency is very good produced about $95.14 \%$ recognition rate. This is because of the detection scheme that compares only the required features which are being trained according to the specific type of defects. Another advantage of the system is its simplicity and ease of use, since the matching algorithm uses a single edge detection method that was built to process the current environment during training phase instead of predetermined environment setting. This greatly reduces the time used for setting and tuning of the vision system whenever there are any changes. Not only that, this system provides an additional data such as height, length and width from the origin of the vision sensor for future usage.

\section{Conclusion}

The aim of this paper is to present a shape based matching vision system for automatic defect detection by using 2 vision sensors as its core. In this paper a concept for a flexible ROI creation visual system was presented where the parameters and characteristics can be easily determined by the user. The proposed visual algorithm concept is easily adaptable and extendable; hence this program is applicable in most situations as it is seen fit by the user. This innovative approach allows the user to select and adapt the system according to their requirements. Additionally, combine with 2 vision sensors provide the system with more precise location as its fit to $\mathrm{x}$ (width), $\mathrm{y}$ (length) and $\mathrm{z}$ (height) pixel coordinate. This system also introduced with inspection system and combined with correction of defect for further improvement.

\section{Acknowledgement}

We wish to express our gratitude to MOHE, Universiti Teknikal Malaysia Melaka (UTeM) especially for Centre for Robotics and Industrial Automation (CeRIA), Centre of Research and Innovation Management (CRIM) and to Faculty of Engineering Technology from UTeM to give the financial (RAGS/1/2015/TK0/FTK/03/B00114) as well as moral support for complete this project successfully.

\section{References}

[1] Gonzalez, R. C., \& Woods, R. E. (2018). Digital image processing. Pearson.

[2] Gu, C., Lim, J. J., Arbeláez, P., \& Malik, J. (2009). Recognition using regions. Proceedings of the IEEE Conference on Computer Vision and Pattern Recognition, pp. 1030-1037.

[3] Mikolajczyk, K., Zisserman, A., \& Schmid, C. (2003). Shape recognition with edge-based features. Proceedings of the British Machine Vision Conference, pp. 779-788.

[4] Salam, R. A., Talib, A. Z. H., Sumari, P., \& Rodrigues, M. A. (2004). Feature extraction in automatic shape recognition system. Proceedings of the IEEE International Conference on Information and Communication Technologies: From Theory to Applications, pp. 467-468.

[5] Carson, C., Belongie, S., Greenspan, H., \& Malik, J. (2002). Blobworld: Image segmentation using expectation-maximization and its application to image querying. IEEE Transactions on Pattern Analysis and Machine Intelligence, 24(8), 1026-1038.

[6] Berg, A. C., Berg, T. L., \& Malik, J. (2005). Shape matching and object recognition using low distortion correspondences. Proceedings of the IEEE Computer Society Conference on Computer Vision and Pattern Recognition, pp. 26-33.

[7] Teck, L. W., Sulaiman, M., Shah, H. N. M., \& Omar, R. (2010). Implementation of shape-based matching vision system in flexible manufacturing system. Journal of Engineering Science and Technology Review, 3(1), 128-135.

[8] Lim, W. T., Sulaiman, M., \& Shah, H. N. M. (2009). Flexible approach for Region of Interest creation for shape-based matching in vision system. Proceedings of IEEE Innovative Technologies in Intelligent Systems and Industrial Applications, pp. 205-208.

[9] Haniff, H. M., Sulaiman, M., Shah, H. N. M., \& Teck, L. W. (2011) Shape-based matching: Defect inspection of glue process in vision system. Proceedings of the IEEE Symposium on Industrial Electronics and Applications, pp. 53-57.

[10] Harun, M. H., Sulaiman, M., Shah, H. N. M., \& Tunggal, D. (2011) Shape-based matching: Application of edge detection using Harris Point. Proceedings of the International Conference on Robotic Automation System, pp. 1-5.

[11] Xu, X., Zhang, X., Han, J., \& Wu, C. (2008). HALCON application for shape-based matching. Proceedings of the 3rd IEEE Conference on Industrial Electronics and Applications, pp. 2431-2434.

[12] Peng, J. J. (2009). A method for recognition of defects in welding lines. Proceedings of the IEEE International Conference on Artificial Intelligence and Computational Intelligence, pp. 366-369.

[13] Sulaiman, M., Shah, H. N. M., Harun, M. H., Lim, W. T., \& Kazim, M. (2013). A 3D gluing defect inspection system using shape-based matching application from two cameras. International Review on Computers and Software, 8(8), 1997-2004.

[14] Sobral, J. L. (2005). Optimised filters for texture defect detection. Proceedings of the IEEE International Conference on Image Processing, pp. 1-4.

[15] Kumar, A., \& Pang, G. K. (2002). Defect detection in textured materials using Gabor filters. IEEE Transactions on Industry Applications, 38(2), 425-440.

[16] Chan, C. H., \& Pang, G. K. (2000). Fabric defect detection by Fourier analysis. IEEE Transactions on Industry Applications, 36(5), 1267-1276.

[17] Meylani, R., Ertuzun, A., \& Erçil, A. (1996). Texture defect detection using the adaptive two-dimensional lattice filter. Proceedings of the IEEE International Conference on Image Processing, pp. 165-168.

[18] Sulaiman, M., Shah, H. N. M., Harun, M. H., Kazim, M., \& Fakhzan, M. N. (2014). Defect inspection system for shape-based matching using two cameras. Journal of Theoretical and Applied Information Technology, 61(2), 288-297.

[19] Xie, X. (2008). A review of recent advances in surface defect detection using texture analysis techniques. Electronic Letters on Computer Vision and Image Analysis, 7(3), 1-22.

[20] Takahashi, T., Matsugano, O., Ide, I., Mekada, Y., \& Murase, H. (2006). Planning of multiple camera arrangement for object recognition in parametric eigenspace. Proceedings of the IEEE 18th International Conference on Pattern Recognition, pp. 603-606.

[21] Jurie, F., \& Dhome, M. (2002). Real time robust template matching Proceedings of the 13th British Machine Vision Conference, pp. 110. 